\title{
Managing the risk of invasive breast cancer in women at risk for breast cancer and osteoporosis: the role of raloxifene
}

\section{Victor G Vogel}

The University of Pittsburgh Cancer Institute, Magee-Womens Hospital, Pittsburgh, PA, USA
Correspondence: Victor G Vogel

The University of Pittsburgh Cancer Institute, Magee-Womens Hospital, 300 Halket Street, Pittsburgh, PA I52I3, USA

$\mathrm{Tel}+\mid$ 4I2 64I 6500

Fax + I 4I2 64I 646I

Email vvogel@magee.edu

\begin{abstract}
Raloxifene hydrochloride is a selective estrogen receptor modulator (SERM) that has antiestrogenic effects on breast and endometrial tissue and estrogenic effects on bone, lipid metabolism, and blood clotting. Raloxifene significantly improves serum lipids and serum markers of cardiovascular disease risk, but it has no significant effect on the risk of primary coronary events. A meta-analysis of randomized, double-blind, placebo-controlled trials of raloxifene for osteoporosis showed the odds of fracture risk were 0.60 (95\% confidence interval $[\mathrm{CI}]=0.49-0.74$ ) for raloxifene $60 \mathrm{mg}$ /day compared with placebo. During 8 years of follow-up in an osteoporosis trial, the raloxifene group had a $76 \%$ reduction in the incidence of invasive ER-positive breast cancer compared with the placebo group. In the STAR trial, the incidence of invasive breast cancer was 4.30 per 1000 women-years with raloxifene and 4.41 per 1000 with tamoxifen; $\mathrm{RR}=1.02 ; 95 \% \mathrm{CI}, 0.82-1.28$. The effect of raloxifene on invasive breast cancer was, therefore, equivalent to that of tamoxifen with more favorable rates of adverse effects including uterine malignancy and clotting events. Millions of postmenopausal women could derive net benefit from raloxifene through reduced rates of fracture and invasive breast cancer.
\end{abstract}

Keywords: raloxifene, osteoporosis, breast cancer risk reduction

\section{Introduction}

Raloxifene hydrochloride is a selective estrogen receptor modulator (SERM) that has antiestrogenic effects on breast and endometrial tissue and estrogenic effects on bone, lipid metabolism, and blood clotting (Vogel 2007). Tamoxifen is the prototypical SERM and reduces the risk of both in situ and invasive breast cancers by half when compared with placebo (Riggs and Hartmann 2003; Swaby et al 2007). The limitations on the use of tamoxifen for breast cancer risk reduction relate to its well-known, but rare, side effects. A number of clinical trials established the benefit of raloxifene on osteoporosis and fracture. Raloxifene significantly improves serum lipids and serum markers of cardiovascular disease risk, but it has no significant effect on the risk of primary coronary events. In the several osteoporosis trials and one heart disease trial, raloxifene decreased the risk of estrogen receptor-positive breast cancer by $44 \%$ to $90 \%$. In the STAR trial, the effect of raloxifene on invasive breast cancer was equivalent to that of tamoxifen with more favorable effects on uterine malignancy and clotting events. Symptomatic side effects are acceptable. In total, the available data indicate that raloxifene represents an acceptable alternative to tamoxifen for the reduction of the risk of postmenopausal breast cancer in high-risk women. The potential market for a compound shown to reduce the risk of breast cancer in postmenopausal women who are at increased risk for breast cancer is more than 10 million women in the US alone. 


\section{Rationale for raloxifene and overview of pharmacology}

Raloxifene hydrochloride is a SERM that has both antiestrogenic and estrogenic effects on breast and endometrial tissue and estrogenic effects on bone, lipid metabolism, and blood clotting (Bryant et al 1996; Delmas et al 1997; Grese et al 1996; Sato et al 1994). It is a benzothiophene with characteristics similar to but distinct from the triphenlyethylene SERMs such as tamoxifen.

In vitro, raloxifene binds to both the alpha and beta subtypes of the estrogen receptor (ER $\alpha$ and $E R \beta$, respectively) (Clemett and Spencer 2000). Compared with 17 $\beta$-estradiol, raloxifene has a relative binding affinity of $46 \%$ at the human $\mathrm{ER} \alpha$ and $26 \%$ at the rat ER $\beta$. In functional transactivation studies, raloxifene antagonizes 17 $\beta$-estradiol-induced estrogen response element (ERE)-mediated activity via both $\mathrm{ER} \alpha$ and ER $\beta$. These distinct ER-ligand conformations appear to recruit different coactivators and corepressors onto the promoter of a target gene by differential protein-protein interactions at the receptor surface. Raloxifene acts as a partial agonist in bone but does not stimulate endometrial proliferation in postmenopausal women, presumably due to some combination of differential expression of transcription factors in the two tissues and the effects of this selective estrogen receptor modulator on ER conformation (Jordan 2006).

\section{Efficacy and comparative studies in osteoporosis}

During the past decade, a number of clinical trials have been conducted to assess the benefit of raloxifene on osteoporosis and fracture. After the publication of the results of the Breast Cancer Prevention Trial (BCPT) (Fisher et al 1998, 2005), these osteoporosis trials reported data related to the incidence of invasive breast cancer among women taking raloxifene compared to those taking placebo. These trials will be reviewed here, emphasizing their designs and the reported effects of raloxifene on both osteoporosis and the incidence of invasive breast cancer.

\section{The MORE Trial}

The Multiple Outcomes of Raloxifene Evaluation (MORE) trial was a multicenter, randomized, double-blind trial, in which women taking raloxifene or placebo were followed for a median of 40 months, from 1994 through 1998, at 180 clinical centers composed of community settings and medical practices in 25 countries, mainly in the US and Europe (Cummings et al 1999, 2002; Ettinger et al 1999;
Cauley et al 2001; Lippman et al 2001; Grady et al 2004). Participants were a total of 7705 postmenopausal women with osteoporosis who were younger than 81 years (mean age, 66.5 years). They were randomly assigned to receive raloxifene, $120 \mathrm{mg}$, raloxifene $60 \mathrm{mg} / \mathrm{day}$, or placebo.

\section{The CORE Trial}

The Continuing Outcomes Relevant to Evista (CORE) trial was conducted to examine the effect of 4 additional years of raloxifene therapy on the incidence of invasive breast cancer in women in MORE who agreed to continue in CORE (Martino 2004; Lippman 2006). 3510 women who had been randomly assigned to receive raloxifene (either 60 or $120 \mathrm{mg}$ /day) in MORE were assigned to receive raloxifene $60 \mathrm{mg} /$ day in CORE. Women who had been assigned to receive placebo in MORE continued on placebo in CORE $(n=1703)$.

\section{Effects on osteoporosis}

After 36 months of the evaluation in 6828 women in the MORE trial, $7.4 \%$ had at least one new vertebral fracture, including $10.1 \%$ of women receiving placebo, $6.6 \%$ of those receiving $60 \mathrm{mg} /$ day of raloxifene, and $5.4 \%$ of those receiving $120 \mathrm{mg} /$ day (Ettinger 1999). The risk of vertebral fracture was reduced $30 \%-50 \%$ in both study groups receiving raloxifene. The frequency of vertebral fracture was reduced both in women who did and did not have prevalent fracture, but the risk of non-vertebral fractures was not significantly reduced by raloxifene. Compared with placebo, raloxifene increased bone mineral density in the femoral neck by about $2 \%$ and in the spine by $2.6 \%$ ( $<<0.001$ for all comparisons). Therefore, in postmenopausal women with osteoporosis, raloxifene increased bone mineral density in the spine and femoral neck and reduced risk of vertebral fracture. There was no demonstrated effect on the risk of hip fracture, and no further reduction in the incidence of vertebral fractures after 4 years of therapy over that seen in the first 3 years (Delmas et al 2002).

A post-hoc analysis of the MORE osteoporosis treatment trial assessed the risk-benefit profile of raloxifene in 7705 postmenopausal women (Barrett-Connor 2004). A major disease outcomes global index resulted in annual rates of $1.39 \%$ and $1.83 \%$ in the raloxifene and placebo groups, respectively (hazard ratio [HR], 0.75; 95\% CI, 0.62-0.92), compatible with a favorable risk-benefit profile for raloxifene for treating postmenopausal osteoporosis. In the RUTH trial (Barrett-Connor 2006) raloxifene reduced the risk of clinical vertebral fractures (HR $=0.65$; 95 percent CI, 0.47 to 0.89 ; absolute risk reduction, 1.3 per 1000). 
A meta-analysis of all randomized, double-blind, placebo-controlled trials has been published and was performed to determine whether the reduction in the risk for vertebral fracture reported with raloxifene was consistent among studies, and to define more accurately the point estimate of the odds ratio (Seeman 2006). Three osteoporosis prevention studies (Jolly 2003), two arms of the MORE trial (Ettinger 1999), and two additional treatment studies (Morii 2003; Lufkin 1998) in which fracture data were available from prospectively scheduled spinal radiographs were included in the analysis. The effects of raloxifene $60 \mathrm{mg} /$ day and $120 \mathrm{mg}$ /day pooled with $150 \mathrm{mg}$ /day were analyzed using an intention to treat analysis to minimize biases. There was no significant heterogeneity among the studies included in the meta-analysis. Odds ratio estimates for the reduction of fracture risk were 0.60 (95\% confidence interval $[\mathrm{CI}]=0.49-0.74)$ for raloxifene $60 \mathrm{mg} /$ day and $0.51(95 \%$ CI $0.41-0.64$ ) for raloxifene $120 \mathrm{mg} /$ day and $150 \mathrm{mg} /$ day. These data indicate that raloxifene consistently reduces the risk of vertebral fracture in postmenopausal women.

Despite these data, some experts consider raloxifene to be second-line therapy (after bisphosphonates and calcium) to be used only in younger postmenopausal women with vertebral osteoporosis given its lack of efficacy in preventing hip fracture (Poole 2006).

\section{The RUTH Trial}

Raloxifene therapy has been associated with improvement in the levels of serum lipoprotein cholesterol, fibrinogen, and homocysteine. The favorable effect of raloxifene on markers of cardiovascular risk, coupled with evidence from observational studies that treatment with estrogen was associated with a reduced risk of coronary heart disease (CHD) in postmenopausal women, led to the design of the Raloxifene Use for The Heart (RUTH) trial to determine the effect of raloxifene on clinical coronary events (Barrett-Connor et al 2006). In the trial, 10,101 postmenopausal women (mean age 67.5 years) with CHD or multiple risk factors for CHD were randomly assigned either to $60 \mathrm{mg}$ of raloxifene daily or placebo and were followed for a median of 5.6 years. The two primary outcomes were coronary events (ie, death from coronary causes, myocardial infarction, or hospitalization for an acute coronary syndrome) and invasive breast cancer. There was no increase in cardiovascular disease including coronary events (myocardial infarction, unstable angina, or coronary ischemia) or cerebrovascular events (stroke or transient ischemic attack) in the osteoporosis treatment trials that compared raloxifene with placebo (Barrett-Connor 2002).
The breast cancer outcome data from the RUTH trial are described below.

\section{Efficacy and comparative studies in breast cancer risk reduction}

The clinical trials that looked at invasive breast cancer as an endpoint are summarized in Table 1.

\section{MORE Trial}

In the MORE trial (Cummings et al 1999, et al 2002; Ettinger et al 1999; Cauley et al 2001; Lippman et al 2001; Grady et al 2004), 13 cases of breast cancer were confirmed among the 5129 women assigned to raloxifene vs 27 among the 2576 women assigned to placebo $(\mathrm{RR}=0.24$; 95\% CI $=0.13-0.44 ; \mathrm{p}<0.001)$. To prevent 1 case of breast cancer, 126 women would need to be treated in this older population of low to average-risk women. Raloxifene decreased the risk of estrogen receptor-positive breast cancer by $90 \%$ (RR, $0.10 ; 95 \%$ CI, 0.04-0.24), but not estrogen receptor-negative invasive breast cancer.

Pre-specified subgroups in the MORE trial were defined by age ( $\geq 65$ versus $<65$ years), age at menopause ( $\geq 9$ vs $<49$ years), body mass index ( $\geq 25 \mathrm{vs}<25 \mathrm{~kg} / \mathrm{m}^{2}$ ), family history of breast cancer, serum estradiol level (5-10 vs $<5,>10$ vs $<5 \mathrm{pmol} / \mathrm{L})$, prior estrogen therapy, and bone mass at baseline entry into the MORE trial, and 5-year predicted risk of breast cancer that was assessed using the modified Gail model. In the placebo group, older age, higher estradiol level, and a family history of breast cancer were associated with an increased breast cancer risk $(\mathrm{p}<0.05)$. Raloxifene therapy was associated with a reduced breast cancer risk in both women at lower and those at higher breast cancer risk. The therapy by family history interaction was significant $(\mathrm{p}=0.04)$ indicating a greater benefit in those women reporting a history of breast cancer in their first-degree relatives.

\section{CORE Trial}

During the 4 years of the CORE trial, there were 61 cases of breast cancer (30 in the placebo group and 31 in the raloxifene group) that were confirmed by adjudication (Martino et al 2004; Lippman et al 2006). Of the 61 breast cancer cases, 52 cases ( 28 in the placebo group and 24 in the raloxifene group) were classified as invasive breast cancer. Women in the raloxifene group had a 59\% reduction in the incidence of invasive breast cancer compared with women in the placebo group (2.1 versus 5.2 cases per 1000 woman-years; $\mathrm{HR}=0.41,95 \% \mathrm{CI}=0.24-0.71$ ). 
Table I Breast cancer risk reduction in studies with raloxifene

\begin{tabular}{|c|c|c|c|c|}
\hline Study & MORE & CORE & RUTH & STAR \\
\hline $\begin{array}{l}\text { Number of women taking } \\
\text { raloxifene }\end{array}$ & 5129 & 3570 & 5044 & 9745 \\
\hline $\begin{array}{l}\text { Number of women in the } \\
\text { comparison group }\end{array}$ & 2576 & 1703 & 5057 & 9726 \\
\hline Comparison drug & Placebo & Placebo & Placebo & Tamoxifen \\
\hline Mean age at study entry & 66.5 & 66.2 & 67.5 & 58.5 \\
\hline Average follow-up time & 40 months & 48 months & 5.6 years & 47 months \\
\hline $\begin{array}{l}\text { No of breast cancers in the } \\
\text { raloxifene group }\end{array}$ & 13 & 40 & 40 & 168 \\
\hline $\begin{array}{l}\text { Event rate in the raloxifene } \\
\text { group (per } 1000 \text { woman- } \\
\text { years) }\end{array}$ & 0.9 & 1.4 & 1.5 & 4.4 \\
\hline $\begin{array}{l}\text { No of breast cancers in the } \\
\text { comparison group }\end{array}$ & 27 & 58 & 70 & 163 \\
\hline $\begin{array}{l}\text { Event rate in the comparison } \\
\text { group (per } 1000 \text { woman- } \\
\text { years) }\end{array}$ & 3.6 & 4.2 & 2.7 & 4.3 \\
\hline $\begin{array}{l}\text { Risk reduction (hazard } \\
\text { rate or risk ratio) and } 95 \% \\
\text { confidence interval }\end{array}$ & $(0.13-0.44)$ & $(0.22-0.50)$ & $\begin{array}{l}0.56 \\
(0.38-0.83)\end{array}$ & $\begin{array}{l}\text { Not } \\
\text { applicable } \\
\text { (no placebo } \\
\text { group) }\end{array}$ \\
\hline
\end{tabular}

Reprinted with permission from Vogel VG. 2007. Raloxifene: a second-generation selective estrogen receptor modulator for reducing the risk of invasive breast cancer in postmenopausal women. Women's Health, 3:139-53. Copyright (c) Future Medicine Ltd.

The ER status was determined for 46 of the 52 cases of invasive breast cancer; 36 cases $(78 \%)$ were ER positive. Women in the raloxifene group had a $66 \%$ reduction in the incidence of invasive ER-positive breast cancers compared with women in the placebo group (1.3 vs 3.9 cases per 1000 woman-years; $\mathrm{HR}=0.34,95 \% \mathrm{CI}=0.18-0.66)$. By contrast, the incidence of invasive ER-negative breast cancer in women who received raloxifene was not different from that in women who received placebo. The overall incidence of breast cancer, regardless of invasiveness, was reduced by $50 \%$ in the raloxifene group compared with the placebo group (2.7 vs 5.5 cases per 1000 woman-years; $\mathrm{HR}=0.50$, $95 \% \mathrm{CI}=0.30-0.82)$.

For the 7705 MORE participants, the total number of reported breast cancers confirmed by adjudication from randomization in MORE to the end of their participation in either MORE or CORE was 121 (56 cancers in the raloxifene group and 65 cancers in the placebo group). During these 8 years, 40 invasive breast cancers were reported in the raloxifene group ( 1.4 cases per 1000 woman-years) and 58 invasive breast cancers were reported in the placebo group (4.2 cases per 1000 woman-years).

The raloxifene group, therefore, had a $66 \%$ reduction in the incidence of invasive breast cancer compared with the placebo group $(\mathrm{HR}=0.34,95 \% \mathrm{CI}=0.22-0.50)$. ER status was determined for 88 cases, and $75 \%$ of these were ER positive. During these 8 years, the raloxifene group had a $76 \%$ reduction in the incidence of invasive ER-positive breast cancer compared with the placebo group ( 0.8 vs 3.2 cases per 1000 woman-years; $\mathrm{HR}=0.24 ; 95 \% \mathrm{CI}=0.15-0.40)$. There was no difference in the incidence rates of invasive ER-negative breast cancer between the raloxifene group and the placebo group. There was no statistically significant difference in the incidence of noninvasive breast cancers reported in the two treatment groups (16 cases for the raloxifene group vs 7 cases for the placebo group; $\mathrm{HR}=1.12,95 \% \mathrm{CI}=0.46-2.73$; $\mathrm{p}=0.80$ ). During the 8 years of the MORE and CORE trials, the overall incidence of breast cancer, regardless of invasiveness, was reduced by $58 \%$ in the raloxifene group compared with the placebo group $(\mathrm{HR}=0.42,95 \% \mathrm{CI}=0.29-0.60$; $\mathrm{P}<0.001)$.

\section{RUTH Trial}

In the RUTH trial (Barrett-Connor et al 2006), raloxifene reduced the risk of invasive breast cancer in lower risk, older women by 44 percent $(\mathrm{HR}=0.56$; $95 \% \mathrm{CI}=0.38-0.83$ ). Similar to the findings in the MORE and CORE trials, raloxifene primarily reduced the incidence 
of estrogen-receptor-positive invasive breast cancer: the absolute risk reduction per 1000 women treated with raloxifene for one year was 1.2 cases of estrogen-receptor-positive invasive breast cancer.

\section{The STAR Trial}

To compare the relative effects and safety of raloxifene and tamoxifen on the risk of developing invasive breast cancer and other disease outcomes, the NSABP conducted the Study of Tamoxifen and Raloxifene (STAR) trial, a prospective, double-blind, randomized clinical trial that began July 1, 1999 in nearly 200 clinical centers throughout North America (Land et al 2006; Vogel 2006). Patients were 19,747 postmenopausal women of mean age 58.5 years with increased 5-year breast cancer risk (mean risk, $4.03 \pm 2.17 \%$ ) as estimated by the Gail model. Participants were randomly assigned to receive either tamoxifen at a dose of $20 \mathrm{mg}$ /day or raloxifene $60 \mathrm{mg}$ /day over 5 years. Outcomes of interest were incidence of invasive breast cancer, uterine cancer, noninvasive breast cancer, bone fractures, and thromboembolic events. The trial was designed to assess statistical equivalence of the two therapies and was powered to report data when 327 cases of invasive breast cancer occurred.

After a median of 3.2 years of therapy in the STAR trial (Vogel 2006), there were 163 cases of invasive breast cancer in women assigned to tamoxifen and 168 in those assigned to raloxifene (incidence, 4.30 per 1000 vs 4.41 per 1000 ; $\mathrm{RR}=1.02 ; 95 \% \mathrm{CI}, 0.82-1.28)$. The cumulative incidence through 72 months for the 2 treatment groups was 25.1 and 24.8 per 1000 for the tamoxifen and raloxifene groups, respectively $(p=0.83)$. When the treatment groups were compared by baseline categories of age, history of LCIS, history of atypical hyperplasia, Gail model 5-year predicted risk of breast cancer (Gail et al 1989; Gail and Costantino 2001), and the number of relatives with a history of breast cancer, the pattern of no differential effect by treatment assignment remained consistent. There were no differences between the treatment groups in regard to distributions by tumor size, nodal status, or estrogen receptor level.

There were fewer cases of noninvasive breast cancer in the tamoxifen group (57 cases) than in the raloxifene group (80 cases) (incidence, 1.51 vs 2.11 per 1000; RR, 1.40; 95\% CI, 0.98-2.00). Cumulative incidence through 6 years was 8.1 per 1000 in the tamoxifen group and 11.6 in the raloxifene group. About $36 \%$ of the cases were lobular carcinoma in situ (LCIS) and 54\% were ductal carcinoma in situ (DCIS), with the balance being mixed types. The pattern of fewer cases among the tamoxifen group was evident for both LCIS and DCIS.

\section{Safety and tolerability Effects on cardiovascular events}

The effect of raloxifene on the incidence of cardiovascular adverse events in postmenopausal women has been reported in the MORE and CORE trials (Dayspring et al 2006; Ensrud et al 2006). Of the 7705 participants originally enrolled in MORE, 4011 were enrolled in CORE. The 8 -year incidence of serious cardiovascular adverse events did not differ significantly between the raloxifene (5.5\%) and placebo $(4.7 \%)$ groups $(\mathrm{HR}=1.16,95 \% \mathrm{CI}=0.86-1.56)$. Similar results were obtained when coronary $(\mathrm{HR}=1.22$, 95\% CI 0.82-1.83) or cerebrovascular $(\mathrm{HR}=1.19,95 \% \mathrm{CI}$ 0.78-1.84) events were analyzed separately, and when cardiovascular events were analyzed in the 459 MORE-CORE participants who were at increased risk of cardiovascular events using previously established criteria (HR 1.03, 95\% CI 0.58-1.82). Thus, there was no evidence of either a beneficial or harmful effect of raloxifene on the incidence of cardiovascular events overall, or coronary or cerebrovascular events, in postmenopausal osteoporotic women who were at relatively low risk of cardiovascular events.

Consistent with these results, raloxifene does not appear to cause adverse effect on lipids and lipoproteins. A post-hoc analysis reported the effects of raloxifene on lipids and lipoproteins in 2659 women with either normal ( $\leq 150 \mathrm{mg} / \mathrm{dL})$ or high $(>150 \mathrm{mg} / \mathrm{dL})$ triglyceride levels from a substudy of the MORE trial (Walsh et al 1998). In both triglyceride subgroups, raloxifene significantly improved low-density lipoprotein cholesterol, total cholesterol, non-high-density lipoprotein cholesterol (HDL-C), apolipoprotein B, apolipoprotein A-I, and fibrinogen compared with placebo $(\mathrm{p}<0.05)$. After raloxifene treatment, women with high triglycerides experienced an equal or greater reduction in cholesterol, lipoprotein parameters, and ratios of total cholesterol to HDL-C and non-HDL-C to HDL-C than were observed in women with normal triglycerides. Mean levels of low-density L-C were reduced by $16.5 \%$ and apolipoprotein B by $15.8 \%$ in women with high triglycerides, and by $12.7 \%$ and $11.3 \%$, respectively, in women with normal triglycerides. These findings substantiate that raloxifene improves concentrations of both cholesterol and beta-lipoprotein. The subgroup of women with high triglycerides, who have elevated cardiovascular risk, appear to derive at least equal, if not greater, overall effect on lipid and lipoprotein lowering with raloxifene. These are important safety considerations 
when evaluating raloxifene for a potential breast cancer risk reduction indication.

In the MORE trial, raloxifene increased the risk of venous thromboembolic disease (RR, 3.1; 95\% CI, 1.56.2), but did not increase the risk of endometrial cancer (RR, 0.8; 95\% CI, 0.2-2.7). During the CORE trial, the relative risk of thromboembolism in the raloxifene group (2.9 events per 1000 woman-years) compared with those in the placebo group (1.3 events per 1000 woman-years) was $2.17(95 \% \mathrm{CI}=0.83-5.70)$ for the CORE enrollees and $3.11(95 \% \mathrm{CI}=0.92-10.44)$ for the $3200 \mathrm{CORE}$ enrollees who resumed taking study medication during the CORE trial. During the 8 years of the MORE and CORE trials, the incidence rate for venous thromboembolic events was 2.2 and 1.3 events per 1000 woman-years for the raloxifene and placebo groups, respectively. During the CORE trial, no cases of pulmonary embolism were reported in the placebo group, compared with nine cases reported in the raloxifene group $(\mathrm{p}=0.066)$. During the 8 years from randomization in MORE until the end of the CORE trial, two women assigned to placebo $(0.16 \%)$ and 17 women assigned to raloxifene $(0.62 \%)$ developed a pulmonary embolism $(p=0.048)$. One death due to pulmonary embolism was reported in the raloxifene group during the 4 years of the CORE trial.

\section{RUTH Trial}

In the RUTH trial, 4 common adverse events (an acute coronary syndrome, anxiety, constipation, and osteoporosis) were reported more frequently in the placebo group than in the raloxifene group, and 7 symptoms or adverse events (ie, arthritis, cholelithiasis, dyspepsia, hot flushes, intermittent claudication, muscle spasm, and peripheral edema) were reported more frequently in the raloxifene group than in the placebo group. Hot flushes, leg cramps, peripheral edema, and gallbladder disease were all statistically significantly more common in women assigned to raloxifene than to placebo. The rates of cholecystectomy did not differ significantly between the treatment groups $(p=0.25)$. The incidences of endometrial cancer and all cancers other than breast cancer did not differ significantly between treatment groups.

Although there was no significant difference in the rates of death from any cause or total stroke according to group assignment, raloxifene was associated with an increased risk of fatal stroke ( 59 vs 39 events; HR = 1.49; 95\% CI, 1.00-2.24; absolute risk increase, 0.7 per 1000 woman-years) and venous thromboembolism ( $\mathrm{HR}=1.44$; 95\% CI, 1.06-1.95; absolute risk increase, 1.2 per 1000 woman-years).
The RUTH trial did not incorporate a global index that weighed risks and benefits as was reported in the BCPT (Fisher et al 1998), but comparison of the absolute reductions and increases in individual end points allows assessment of the net risk-benefit balance (Barrett-Connor et al 2006). The rate of invasive breast cancer was reduced $44 \%$ and translates to 1.2 fewer cancers per 1000 women treated with raloxifene per year among low-risk, older postmenopausal women. The rate of clinical vertebral fractures was 3.7 per 1000 women per year, and the absolute reduction was 1.3 fractures per 1000 women-years. These two benefits came at a cost of 1.2 more cases of venous thromboembolism and 0.7 excess fatal strokes per 1000 women-years. For women in the RUTH cohort that comprised individuals with or at increased risk for coronary heart disease, the moderate benefits of raloxifene for breast cancer risk reduction appeared to some observers not to justify the risks (Stefanick 2006).

\section{Quality of life, patient satisfaction, acceptability, adherence, and uptake}

In the STAR trial, patient-reported symptoms were collected from all participants using a 36-item symptom checklist (Land et al 2006). Quality of life was measured with the Medical Outcomes Study Short-Form Health Survey (SF-36) (McHorney et al 1992, 1993, 1994; Ware and Sherbourne 1992), the Center for Epidemiologic Studies-Depression (CES-D) (Radloff 1977), and the Medical Outcomes Study Sexual Activity Questionnaire (Sherbourne 1992) in a substudy of 1983 participants with a median follow-up of 5.4 years (range, 4.6-6.0 years). Questionnaires were administered before treatment, every 6 months for 60 months and at 72 months. Primary quality of life end points were the SF-36 physical (PCS) and mental (MCS) component summaries.

Among women in the quality of life analysis in STAR, mean PCS, MCS, and CES-D scores worsened modestly throughout the study with no significant difference between the tamoxifen and raloxifene groups. Sexual function was slightly better for participants assigned to tamoxifen. Of the women in the symptom assessment analyses, those in the raloxifene group reported greater mean symptom severity over 60 months of assessments than the women in the tamoxifen group for musculoskeletal problems, dyspareunia, and weight gain. Women in the tamoxifen group reported greater mean symptom severity for gynecological problems, vasomotor symptoms, leg cramps, and bladder control symptoms. No significant differences existed, however, between the tamoxifen and raloxifene groups in patient-reported outcomes for physical health, mental health, and depression, although the tamoxifen group reported better sexual 
function. Although mean symptom severity was low among these postmenopausal women, those in the tamoxifen group reported more gynecological problems, vasomotor symptoms, leg cramps, and bladder control problems, whereas women in the raloxifene group reported more musculoskeletal problems, dyspareunia, and weight gain.

There were 36 cases of uterine cancer with tamoxifen and 23 with raloxifene (RR, 0.62; 95\% CI, 0.35-1.08). No differences were found for other invasive cancer sites, for ischemic heart disease events, or for stroke.

Thromboembolic events (ie, pulmonary embolism and deep venous thrombosis) occurred less often in the raloxifene group (RR, 0.70; 95\% CI, 0.54-0.91). The absolute rate of venous thromboembolism was significantly lower among women assigned to raloxifene (2.6 per 1000) than among those assigned to tamoxifen (3.7 per 1000). The cumulative incidence of serious clotting events at 6 years was 21.0 per 1000 for the raloxifene group and 16.0 per 1000 for raloxifene group. Pulmonary embolism and DVT occurred in 54 vs 35 women $(\mathrm{RR}=0.64 ; 95 \% \mathrm{CI}, 0.41-1.00)$ and in 87 vs 65 women $(\mathrm{RR}=0.74 ; 95 \% \mathrm{CI}, 0.53-1.03)$ assigned to tamoxifen and raloxifene, respectively.

There were 53 strokes associated with tamoxifen in the STAR trial and 51 stroke events among the women taking raloxifene. The number of osteoporotic fractures in the groups was similar. There were fewer cataracts $(R R$, 0.79; 95\% CI, 0.68-0.92) and cataract surgeries (RR, 0.82; 95\% CI, 0.68-0.99) in the women taking raloxifene. There was no difference in the total number of deaths or in causes of death.

\section{Conclusions}

The potential market for a compound shown to reduce the risk of invasive breast cancer in postmenopausal women who are at risk for breast cancer is large (Freedman et al 2003). Of the more than 65 million women aged 35-79 without reported breast cancer in the US in 2000, more than 10 million women (15.5\%) would be eligible for tamoxifen chemoprevention using the eligibility criteria of the BCPT. In the US, there are more than 33 million postmenopausal women, and 9 million of these women are eligible for risk reduction with a SERM. Of the 50 million white US women aged 35-79, more than 2 million, or about $5 \%$, would have a positive benefit/risk index for SERM-based chemoprevention. An estimate of the net benefit among non-white women is difficult due to the lack of these women in the clinical trials. Efforts are ongoing to increase participation of non-white women in risk reduction clinical trials. Among the postmenopausal women, 1.2 million women would derive net benefit from tamoxifen. Among white women in North America, more than 28,000 breast cancers would be prevented or deferred if those women who have a positive net benefit index took tamoxifen over the next 5 years. A similar number of women in Europe could also derive benefit from chemoprevention of breast cancer with a SERM. Estimates for the rest of the world are not available. Approximately 500,000 women worldwide are currently taking raloxifene for the management of osteoporosis.

Raloxifene is approved by both the European Union and the US Food and Drug Administration (FDA) for the prevention and treatment of osteoporosis in postmenopausal women. Based on the extensive clinical experience reported to date, the FDA approved raloxifene to reduce the risk of invasive breast cancer in high-risk, postmenopausal women in September 2007. These women would include those with high quantitative risks for breast cancer, those with lobular carcinoma in situ or atypical ductal or lobular hyperplasia, and in women with a history of breast cancer in a first-degree female relative (mother, sister, or daughter).

The findings from the MORE and CORE trials suggest that raloxifene therapy is associated with a reduced risk of invasive breast cancer in postmenopausal women irrespective of the presence or absence of risk factors, but that its effect are greater in women with a family history of breast cancer. The cumulative results from all of the trials considered together indicate that both absolute benefits and absolute risks will vary depending on the risk profiles of women receiving treatment. Raloxifene does not significantly affect the risk of cardiac outcome events despite its known favorable effects on serum lipids. In addition, in the older population of women in RUTH who were at low to average risk of breast cancer, the benefits of raloxifene in reducing the risks of invasive breast cancer and vertebral fracture were negatively balanced by the increased risks of venous thromboembolism and fatal stroke. The small difference ( 0.6 per 1000 woman-years) in the rate of non-invasive breast cancer reported in the STAR trial when comparing tamoxifen with raloxifene will have a small impact on the estimate of the net benefit of raloxifene for reducing the risk of breast cancer given that non-invasive breast cancer is not a life-threatening event and should not diminish the perceived utility of raloxifene for this indication.

Raloxifene is a unique SERM with distinct activity and toxicity profiles. Extensive experience from prospective investigations has established its safety and efficacy in the management of postmenopausal osteoporosis. Although it has no apparent beneficial effect on CHD, it has no adverse 
effect either. Three prospective clinical trials have established its benefit in reducing the risk of invasive breast cancer, and it offers safety advantages when compared with tamoxifen in postmenopausal women who are at increased risk for breast cancer. Symptomatic side effects are acceptable as reported in the large, prospectively blinded clinical populations summarized here and followed during years of raloxifene administration. The risk of other cancers, fractures, ischemic heart disease, and stroke are similar for both raloxifene and tamoxifen. Raloxifene thus offers an alternative to tamoxifen for the reduction of breast cancer risk in high-risk postmenopausal women with a superior risk-benefit profile based upon the benefits and risks reviewed here.

\section{Disclosures}

The author reports no conflicts of interest.

\section{References}

Barrett-Connor E, Grady D, Sashegyi A, et al. 2002. Raloxifene and cardiovascular events in osteoporotic postmenopausal women: four-year results from the MORE (Multiple Outcomes of Raloxifene Evaluation) randomized trial. JAMA, 287:847-57.

Barrett-Connor E, Cauley JA, Kulkarni PM, et al. 2004. Risk-benefit profile for raloxifene: 4-year data from the Multiple Outcomes of Raloxifene Evaluation (MORE) randomized trial. J Bone Miner Res, 19:1270-5.

Barrett-Connor E, Mosca L, Collins P, et al. 2006. Raloxifene Use for The Heart (RUTH) Trial Investigators. Effects of raloxifene on cardiovascular events and breast cancer in postmenopausal women. N Engl J Med, 355:125-37.

Bryant HU, Glasebrook AL, Yang NN, et al. 1996. A pharmacological review of raloxifene. J Bone Miner Metab, 14:1-9.

Cauley JA, Norton L, Lippman ME, et al. 2001. Continued breast cancer risk reduction in postmenopausal women treated with raloxifene: 4-year results from the MORE trial. Breast Cancer Res Treat, 65:125-34.

Clemett D, Spencer CM. 2000. Raloxifene: a review of its use in postmenopausal osteoporosis. Drugs, 60:379-411.

Cummings SR, Eckert S, Krueger KA, et al. 1999. The effect of raloxifene on risk of breast cancer in postmenopausal women: results from the MORE randomized trial. JAMA, 281:2189-97.

Cummings SR, Duong T, Kenyon T, et al. 2002. Serum estradiol level and risk of breast cancer during treatment with raloxifene. JAMA, 287:216-20.

Dayspring T, Qu Y, Keech C. 2006. Effects of raloxifene on lipid and lipoprotein levels in postmenopausal osteoporotic women with and without hypertriglyceridemia. Metabolism, 55:972-9.

Delmas PD, Bjarnason NH, Mitlak BH, et al. 1997. Effects of raloxifene on bone mineral density, serum cholesterol concentrations, and uterine endometrium in postmenopausal women. $N$ Engl $\mathrm{J} \mathrm{Med,}$ 337:1641-7.

Delmas PD, Ensrud KE, Adachi JD, et al. 2002. Efficacy of raloxifene on vertebral fracture risk reduction in postmenopausal women with osteoporosis: four-year results from a randomized clinical trial. J Clin Endocrin Metab, 87:3609-617.

Ensrud K, Genazzani AR, Geiger MJ, et al. 2006. Effect of raloxifene on cardiovascular adverse events in postmenopausal women with osteoporosis. Am J Cardiol, 97:520-7.

Ettinger B, Black DM, Mitlak BH, et al. 1999. Reduction of vertebral fracture risk in postmenopausal women with osteoporosis treated with raloxifene: results from a 3-year randomized clinical trial. JAMA, 282:637-45.
Fisher B, Costantino JP, Wickerham L, et al. 1998. Tamoxifen for the prevention of breast cancer: report of the National Surgical Adjuvant Breast and Bowel Project P-1 Study. J Natl Cancer Inst, 90:1371-88.

Fisher B, Costantino JP, Wickerham DL, et al. 2005. Tamoxifen for the prevention of breast cancer: current status of the National Surgical Adjuvant Breast and Bowel Project P-1 study. J Natl Cancer Inst, 97:1652-62.

Freedman AN, Graubard BI, Rao SR, et al. 2003. Estimates of the number of US women who could benefit from tamoxifen for breast cancer chemoprevention. J Natl Cancer Inst, 95:526-32.

Gail MH, Brinton LA, Byar DP, et al. 1989. Projecting individualized probabilities of developing breast cancer for white females who are being examined annually. J Natl Cancer Inst, 81:1879-86.

Gail MH, Costantino JP. 2001. Validating and improving models for projecting the absolute risk of breast cancer. J Natl Cancer Inst, 93:334-5.

Grady D, Ettinger B, Moscarelli E, et al. 2004. Safety and adverse effects associated with raloxifene: Multiple Outcomes of Raloxifene Evaluation. Obstet Gynecol, 104:837-44.

Grese TA, Cho S, Finley DR, et al. 1997. Structure-activity relationships of selective estrogen receptor modulators: modifications to the 2-arylbenzothiophene core of raloxifene. J Med Chem, 40:146-67.

Jolly EE, Bjarnason NH, Neven P, et al. 2003. Prevention of osteoporosis and uterine effects in postmenopausal women taking raloxifene for 5 years. Menopause, 10:337-44.

Jordan VC. 2006. The science of selective estrogen receptor modulators: concept to clinical practice. Clin Cancer Res, 12:5010-3.

Land SR, Wickerham DL, Costantino JP, et al. 2006. Patient-reported symptoms and quality of life during treatment with tamoxifen or raloxifene for breast cancer prevention: the NSABP Study of Tamoxifen and Raloxifene (STAR) P-2 trial. JAMA, 295:2742-51.

Lippman ME, Cummings SR, Disch DP, et al. 2006. Effect of raloxifene on the incidence of invasive breast cancer in postmenopausal women with osteoporosis categorized by breast cancer risk. Clin Cancer Res, 12:5242-7.

Lippman ME, Krueger KA, Eckert S, et al. 2001. Indicators of lifetime estrogen exposure: effect on breast cancer incidence and interaction with raloxifene therapy in the Multiple Outcomes of Raloxifene Evaluation study participants. J Clin Oncol, 19:3111-6.

Lufkin EG, Whitaker MD, Nickelsen T, et al. 1998. Treatment of established postmenopausal osteoporosis with raloxifene: a randomized trial. J Bone Miner Res, 13:1747-54.

Martino S, Cauley JA, Barrett-Connor E, et al. 2004. Continuing outcomes relevant to Evista: breast cancer incidence in postmenopausal osteoporotic women in a randomized trial of raloxifene. J Natl Cancer Inst, 96:1751-61.

McHorney CA, Ware JE Jr, Lu JF, Sherbourne CD. 1994. The MOS 36-item Short-Form Health Survey (SF-36), III: tests of data quality, scaling assumptions, and reliability across diverse patient groups. Med Care, 32:40-66.

McHorney CA, Ware JE Jr, Raczek AE. 1993. The MOS 36-Item Short-Form Health Survey (SF-36), II: psychometric and clinical tests of validity in measuring physical and mental health constructs. Med Care, 31:247-63.

McHorney CA, Ware JE Jr, Rogers W, et al. 1992. The validity and relative precision of MOS short- and long-form health status scales and Dartmouth COOP charts: results from the Medical Outcomes Study. Med Care, 30(5 Suppl):MS253-MS265.

Morii H, Ohashi Y, Taketani Y, et al. 2003. Effect of raloxifene on bone mineral density and biochemical markers of bone turnover in Japanese postmenopausal women with osteoporosis: results from a randomized placebo-controlled trial. Osteoporos Int, 14:793-800.

Poole KES, Compston JE. 2006. Osteoporosis and its management. BMJ, 333:1251-6.

Radloff LS. 1977. The CES-D Scale: a self-report depression scale for research in the general population. Appl Psychol Meas, 1:385-401. 
Riggs BL, Hartmann LC. 2003. Selective estrogen-receptor modulators-mechanisms of action and application to clinical practice. N Engl J Med, 348:618-29.

Sato M, Glasebrook AL, Bryant HU. 1994. Raloxifene: a selective estrogen receptor modulator. J Bone Miner Metab, 12:S9-S20.

Seeman E Crans GG, Diez-Perez A, et al. 2006. Anti-vertebral fracture efficacy of raloxifene: a meta-analysis. Osteoporos Int, 17:313-6.

Sherbourne CD. 1992. Social functioning: sexual problems measures. In: Stewart AL, Ware JE (eds). Measuring functioning and well-being: The Medical Outcomes Study approach. Durham, NC: Duke University Press p. 194-204.

Stefanick ML. 2006. Risk-benefit profiles of raloxifene for women. $\mathrm{NEngl}$ J Med, 355:190-2.

Swaby RF. Sharma CG. Jordan VC. 2007. SERMs for the treatment and prevention of breast cancer. Rev Endocr Metab Disord, 8:229-39.
Vogel VG. 2007. Raloxifene: a second-generation selective estrogen receptor modulator for reducing the risk of invasive breast cancer in postmenopausal women. Women's Health, 3:139-53.

Vogel VG, Costantino JP, Wickerham DL, et al. 2006. National Surgical Adjuvant Breast and Bowel Project (NSABP). Effects of tamoxifen vs raloxifene on the risk of developing invasive breast cancer and other disease outcomes: the NSABP Study of Tamoxifen and Raloxifene (STAR) P-2 trial. JAMA, 295:2727-41.

Walsh BW, Kuller LH, Wild RA, et al. 1998. Effects of raloxifene on serum lipids and coagulation factors in healthy postmenopausal women. JAMA, 279:1445-51.

Ware JE Jr, Sherbourne CD. 1992. The MOS 36-item short-form health survey (SF-36), I: conceptual framework and item selection. Med Care, 30:473-83. 
